\title{
Análisis prospectivo de hernioplastías umbilicales en adultos*
}

\author{
Drs. GERMÁN VISCIDO ${ }^{1}$, DANIEL NAPOLITANO ${ }^{1}$, GUILLERMO RIVOIRA ${ }^{1}$, MATÍAS PARODI ${ }^{1}$, \\ MARTÍN BAROTO ${ }^{1}$, HÉCTOR PICÓN-MOLINA ${ }^{1}$, MARCELO DONIQUIAN ${ }^{1}$, RAFAEL PALENCIA ${ }^{1}$
}

1 Servicio de Cirugía General, Clínica Universitaria Privada Reina Fabiola, Córdoba, Argentina.

Abstract

\section{Umbilical mesh hernia repair. Experience in 60 patients}

Background: There is a lack of consensus on the best technique umbilical for hernia repair and several options have been use. The systematic use of prostheses is currently recommended. Aim: To evaluate the treatment of umbilical hernias with a mesh and assess the placement of a prosthetic cone as an alternative. Materials and Methods: We evaluated prospectively the surgical procedures in 60 patients with umbilical hernia aged 30 to 86 years (40 females). We excluded surgical procedures in which a mesh was not used and those performed as emergencies. When the hernia ring was of less than $3 \mathrm{~cm}$ in diameter, a polypropylene cone or plug was placed. A preperitoneal mesh technique was used when rings were larger. Results: Forty five patients had a ring of less than $3 \mathrm{~cm}$. Among these, a polypropylene cone or plug was placed in 30 and 15 patients, respectively. A mesh was placed in the remaining patients. As surgical complications, one patient had a hematoma (1.6\%) and three patients had seromas $(5 \%)$. There were no surgical site infections or hernia relapse. Conclusions: Umbilical hernia repair using with polypropylene prostheses is a safe and easy to implement technique.

Key words: Umbilical hernia, mesh, polypropylene plug, cone.

\section{Resumen}

Introducción: A pesar de su alta prevalencia, la hernia umbilical no ha sido tan estudiada en la bibliografía como otros defectos herniarios. No hay consenso en la mejor técnica para su reparación y varias opciones han sido utilizadas para el tratamiento de esta enfermedad. Actualmente se recomienda la utilización sistemática de prótesis para su reparación. Objetivo: Evaluar el tratamiento de la hernia umbilical con mallas y valorar la colocación de prótesis en forma de cono como alternativa en la reparación. Diseño: Prospectivo, observacional. Lugar de Aplicación: Clínica Universitaria Privada. Material y Métodos: Evaluamos 60 hernioplastias entre el $1^{\circ}$ de enero del 2005 y $1^{\circ}$ de agosto del 2007. Se excluyeron herniorrafias y cirugías urgentes. En hernias con anillo menor a $3 \mathrm{~cm}$ de diámetro colocamos plug o cono de polipropileno; anillos mayores a $3 \mathrm{~cm}$ se repararon con malla preperitoneal. Resultados: Edad promedio 50 años, sexo

*Recibido el 22 de Septiembre de 2008 y aceptado para publicación el 9 de Octubre de 2008.

Trabajo preliminar presentado durante la Sesión de Temas Libres: "Paredes Abdominales", en el $78^{\circ}$ Congreso Argentino de Cirugía 21 al 24 de Octubre del 2007.

Correspondencia: Dr. Germán R. Viscido

Derqui 312 Piso $6^{\circ} \mathrm{E}$, Ciudad de Córdoba, Argentina

E-mail: germanviscido@hotmail.com 
femenino 40 pacientes, 56 hernias primarias. Encontramos 45 pacientes con anillo menor a $3 \mathrm{~cm}$, colocándose en 30 cono de polipropileno y en 15 plug. El resto de los pacientes tenían anillos de más de $3 \mathrm{~cm}$ y se les colocó una malla preperitoneal. Complicaciones: 1 hematoma (1,6 \%), 3 seromas (5\%). No hubo infecciones del sitio quirúrgico ni recidivas. Conclusiones: La reparación de una hernia umbilical con prótesis de polipropileno es una técnica segura y fácil de aplicar. La colocación de cono de polipropileno, es una técnica que debe ser considerada como alternativa del tapón en hernias con anillo menor a $3 \mathrm{~cm}$.

Palabras clave: Hernia umbilical, malla, plug de polypropyleno, cono.

\section{Introducción}

El ombligo es una de las áreas débiles del abdomen y un sitio común de herniación ${ }^{1}$.

Representa el 6\% de todas las hernias de pared del adulto ${ }^{2}$. A pesar de su alta prevalencia en la población adulta, no ha sido tan estudiada y valorada en la bibliografía como otros defectos herniarios $^{3}$.

No hay consenso en la mejor técnica para la reparación de la hernia umbilical y por lo tanto varias técnicas quirúrgicas como sutura primaria, reparación de Mayo, reparación con malla y cirugía laparoscópica han sido utilizadas para el tratamiento de esta enfermedad ${ }^{1}$. Debido a la alta incidencia de recurrencias que se relatan con las técnicas anatómicas ${ }^{3-6}$, actualmente se recomienda efectuar una reparación sin tensión, utilizando sistemáticamente prótesis en hernias con anillo mayor a $1 \mathrm{~cm}^{7,8}$, incluso en todas las hernias sin importar el tamaño del defecto ${ }^{1}$.

El objetivo primario de nuestro trabajo es evaluar el tratamiento de las hernias umbilicales con la utilización de mallas protésicas.

El objetivo secundario es valorar la colocación de cono de polipropileno, como alternativa para reparación de la hernia umbilical.

\section{Material y Método}

Trabajo prospectivo, observacional. Se analizaron todas las hernioplastias umbilicales programadas realizadas por el Servicio de Cirugía General de la Clínica Universitaria Privada Reina Fabiola de la ciudad de Córdoba, entre el $1^{\circ}$ de enero del 2005 y $1^{\circ}$ de agosto del 2007. Se excluyeron herniorrafias y cirugías urgentes.

Se evaluó: edad y sexo, hernia primaria o recidivada, tipo de anestesia, tamaño del anillo herniario y técnica quirúrgica realizada, duración de la cirugía, estadía hospitalaria, dolor según Escala Visual Análoga, retorno laboral, complicaciones tempranas y tardías, mortalidad y controles posteriores.

En todos los pacientes se realizó antibióticoprofilaxis con Cefalotina 2 gr durante la inducción anestésica o media hora antes del comienzo del acto quirúrgico. En caso de alergia a Penicilina se usó Clindamicina $600 \mathrm{mg}^{9}$.

La analgesia postquirúrgica se realizó con Ketorolac $60 \mathrm{mg}$ al final de la cirugía, una dosis de 30 mg a las 5 horas posteriores. Al alta se utilizó Ibuprofeno $600 \mathrm{mg}$ cada 12 horas durante 3 días.

Se otorga una planilla impresa en la cual figuran indicaciones que debe realizar el paciente para un mejor resultado alejado de la cirugía:

- No conducir personalmente vehículos durante 5 días.

- No realizar gimnasia ni deportes durante 3 semanas.

- No realizar esfuerzos ni práctica de deportes competitivos durante 6 semanas.

- Retomar su baño habitual, sin refregar la herida manteniendo un secado cuidadoso de la misma.

Los pacientes fueron citados a los 14 días del postoperatorio para el retiro de puntos. Se realizaron controles a los 45, 180, 360 y 720 días de la cirugía en consultorio de paredes abdominales a cargo de un miembro del equipo del Servicio de Cirugía General.

Se realizaron 3 técnicas protésicas en los pacientes dependiendo del diámetro del anillo:

En pacientes con anillo menor a $3 \mathrm{~cm}$ de diámetro se utilizaron:

- Colocación de tapón o "plug", confeccionado con malla de polipropileno y suturado a los bordes del anillo umbilical con 4 puntos cardinales de poliamida 0,15 , teniendo la precaución de cerrar el saco en caso de apertura para evitar el contacto de la prótesis con el contenido abdominal.

- Colocación de cono, confeccionado con malla de polipropileno y fijado a los bordes del anillo con puntos sueltos de poliamida 0,15 .

En pacientes con anillo mayor a $3 \mathrm{~cm}$ de diámetro se utilizó:

- Colocación de malla de polipropileno en el espacio preperitoneal, previa disección cuidadosa del mismo. El tamaño de la misma sobrepasó entre 2,5 y $3 \mathrm{~cm}$ los bordes del anillo herniario y se fijó con puntos sueltos de poliamida 0,15 a la aponeurosis sana. 


\section{Resultados}

Durante el período estudiado se realizaron 60 hernioplastías umbilicales electivas. Las características de la población se describen en la Tabla 1.

Al estudiar el tipo de anestesia utilizada 58 $(96,6 \%)$ pacientes fueron operados con bloqueo regional y $2(3,4 \%)$ pacientes con anestesia general.

En $45(75 \%)$ enfermos se encontró un anillo de menos de $3 \mathrm{~cm}$, realizándose en $30(50 \%)$ la colocación de un cono de polipropileno. En 15 (25\%) se colocó un tapón de igual material en el anillo umbilical. Se hallaron 15 pacientes con un anillo de más de $3 \mathrm{~cm}$ en los que se colocó una prótesis de polipropileno en el espacio preperitoneal, fijada con puntos sueltos de poliamida 0,15 .

La duración promedio de la cirugía fue de 39 minutos para la colocación de cono y tapón, en cambio al colocar prótesis en espacio preperitoneal este fue de 60 minutos.

Al analizar la estadía hospitalaria observamos que $18(30 \%)$ enfermos en los que se colocó cono o tapón fueron dados de alta antes de las doce horas del postoperatorio. El resto de los pacientes $(n=27)$ de este grupo fue dado de alta a las 24 horas. Todos los pacientes a los que se colocó malla fueron dados de alta entre las 24 y 48 horas del postoperatorio.

Analizando el dolor posquirúrgico, el 100\% de los enfermos en los que se colocó cono o tapón tuvieron dolor leve. En el grupo en los que se trató con prótesis preperitoneal, el 79\% $(\mathrm{n}=13)$ tuvo dolor leve y sólo 2 pacientes dolor moderado.

En cuanto al retorno laboral el $75 \%$ de los enfermos retornó al trabajo antes de la semana del posquirúrgico, el $10 \%$ estuvo en condiciones pero no lo hizo y el $15 \%$ restante luego de la primera semana. El seguimiento por consultorio varió entre 60 y 930 días.

Las complicaciones postoperatorias y mortalidad se detallan en la Tabla 2.

Durante los controles posteriores que se realizaron en consultorio, sólo 4 pacientes presentaron dolor leve en el segundo control, siendo tratados con analgésicos comunes con buena evolución. En los sucesivos controles no se han encontrado otro tipo de inconvenientes en estos pacientes.

\section{Discusión}

Cerca del $10 \%$ de todas las hernias primarias de la pared abdominal consisten en hernias umbilicales y epigástricas ${ }^{10}$. Su reparación, por lo tanto, representa una tarea importante en cirugía pediátrica y del adulto.

Es una enfermedad relativamente frecuente, que en el $90 \%$ de los casos es un defecto adquirido ${ }^{3,10}$, representando una herniación indirecta a través del canal umbilica ${ }^{10}$. Factores que predisponen a la aparición de este tipo de hernias son: obesidad extrema, historia de múltiples embarazos, ascitis y grandes tumores abdominales ${ }^{3,10}$.

Sorprendentemente, y una de las inquietudes que nos llevó a realizar esta investigación, es que no existe consenso acerca de cuál es la reparación que debería ser realizada. Los ensayos clínicos y los estudios que divulgan experiencias con ciertos procedimientos quirúrgicos son escasos en la literatu$\mathrm{ra}^{10}$. Sólo Utrera González, describe un protocolo de tratamiento de acuerdo al tamaño del anillo umbilical ${ }^{11}$.

En nuestra casuística el sexo femenino fue más frecuente, estos datos concuerdan con la mayoría de la bibliografía consultada ${ }^{1-3}$. Aproximadamente el $7 \%$ de los pacientes tuvieron asociada una hernia epigástrica y el 18\% hernia inguinal bilateral.

Aunque actualmente la anestesia local para la reparación de la hernia umbilical es una técnica aceptada $^{3,4,12-14}$, la mayoría de los pacientes de nuestra serie fueron operados con bloqueo regional sin complicaciones.

La utilización de tapón protésico en la reparación de las hernias umbilicales es una técnica con buenos resultados $3,7,11,12$ en hernias con anillo menor a $3 \mathrm{~cm}$ de diámetro. En cambio la colocación de un cono protésico, como técnica alternativa al ta-
Tabla 1. Características analizadas

\begin{tabular}{ll}
\hline Edad promedio & 50 años $(30-86)$ \\
Sexo & Femenino $40(66,6 \%)$ \\
& Masculino $20(37,8 \%)$ \\
Tipo de hernia & Primaria $56(93,3 \%)$ \\
& Recidivadas 4 (6,7\%) \\
Asociadas a hernia epigástrica & $6(10 \%)$ \\
Asociada a hernia inguinal bilateral & $11(18 \%)$ \\
\hline
\end{tabular}

Tabla 2. Complicaciones postquirúrgicas y mortalidad

\begin{tabular}{llr}
\hline Hematoma & 1 & $(1,6 \%)$ \\
Seroma & 3 & $(5 \%)$ \\
Infección de herida & 0 & \\
Rechazo de prótesis & 0 & \\
Recidivas & 0 & \\
Mortalidad & 0 & \\
\hline
\end{tabular}


pón, no es muy descripta en la literatura hallando sólo un trabajo en lo consultado ${ }^{13}$.

El tiempo quirúrgico promedio de nuestra serie fue de 38 minutos para hernioplastías con anillo menor a $3 \mathrm{~cm}$ y de 60 minutos para la colocación de malla preperitoneal. Este hecho se explica ya que estos defectos son más grandes y para su reparación se necesita labrar un espacio para colocar la malla, lo que requiere mayor tiempo de disección.

En la estadía hospitalaria de los pacientes con tapón y cono puede observarse que un porcentaje no menor de pacientes pueden ser dados de alta durante el mismo día. Aquellos en los que se colocó una prótesis tienen una estadía poco más prolongada debido a la magnitud de la disección quirúrgica, pero menor a la bibliografía consultada ${ }^{1,2}$.

Arroyo et al, en su trabajo aleatorizado en el que compara la reparación con y sin prótesis no encuentra grandes diferencias entre una y otra técnica al analizar las complicaciones posquirúrgicas ${ }^{4}$. El mismo autor en otro trabajo en el que utiliza únicamente mallas protésicas para la reparación informa un 5,6\% de seromas, $2,3 \%$ de hematomas, $1,4 \%$ de infecciones de la herida y $0,95 \%$ de rechazos de la malla ${ }^{3}$. Tampoco Eryilmaz et al, informa diferencias significativas entre las dos técnicas al comparar seromas, hematomas e infección de la herida ${ }^{1}$. Polat et al, utilizando para la reparación "Prolene Hernia System" y comparando con la técnica de Mayo y la colocación "onlay" de una malla tampoco relata diferencias entre los grupos ${ }^{2}$. Todos ellos encuentran un bajo porcentaje de complicaciones al utilizar técnicas protésicas.

Uno de los pilares de la evaluación de una técnica quirúrgica de hernias, quizás el más importante, es el índice de recidivas. Históricamente la recidiva herniaria con la técnica de Mayo oscila entre 20$28 \%{ }^{12}$. Cuando los índices de recidiva de ésta técnica son comparados con los de técnicas protésicas se observa que son significativamente más altos, no superando el $2 \%$ en éstas últimas ${ }^{1,2,4}$. Otros trabajos retrospectivos que comparan herniorrafias con hernioplastías informan $0 \%$ de recidivas con la utilización de mallas ${ }^{14}$.

Creemos que un sesgo de nuestro trabajo es la escasa cantidad de pacientes de la serie. Pensamos que la realización de trabajos prospectivos y aleatorizados con número aceptable de pacientes puede aclarar y definir cual o cuales técnicas deben ser utilizadas para esta patología.

\section{Conclusión}

La reparación de una hernia umbilical con prótesis de polipropileno es una técnica segura y fácil de aplicar. La colocación de cono de polipropileno, es una técnica que debe ser considerada como alternativa del tapón, ya que además de rápida realización, leve dolor postquirúrgico y pronta alta del paciente, presenta escasas complicaciones y nulo índice de recidiva en nuestra experiencia.

\section{Referencias}

1. Eryilmaz R, Sahin M, Tekelioglu M. Wich Repair in Umbilical Hernia of Adults: Primary or Mesh? Int Surg 2006; 91: 258-261

2. Polat C, Dervisoglu A, Senyurek G, Bilgin M, Erzurumlu $\mathrm{K}$, Ozkan K. Umbilical hernia repair with the prolene hernia system. Am J Surg 2005; 190: 61-64.

3. Arroyo A, Costa D, Fernández A, Pérez F, Serrano P, García P y col. ¿ Debe ser la hernioplastia la técnica de elección en el tratamiento de la hernia umbilical del adulto? Cir Esp 2001; 70: 69-71.

4. Arroyo A, García P, Pérez F, Andreu J, Candela F, Calpena R. Randomized clinical trial comparing suture and mesh repair of umbilical hernia in adults. Br J Surg 2001; 88: 1321-1323.

5. García Ureña MA, Rico Selas P, Seoane J, Marcello M, Moreno González E, Corral Sánchez MA y col. Hernia umbilical del adulto. Resultados a largo plazo en pacientes operados de urgencia. Cir Esp 1994; 56: 302-306.

6. Hidalgo M, Higuero F, Álvarez-Caperochipi J, Machuca J, Laporte E, Figueroa J y col. Hernias de la pared abdominal. Estudio multicéntrico epidemiológico (1993-1994). Cir Esp 1996; 59: 309-405.

7. Feliu Palá X, Carbonell Tatay F. "Hernia de la Línea Alba y Anteriores". En: Cirugía AEC: Manual de la Asociación Española de Cirujanos. $1^{\circ}$ Edición. Madrid: Editorial Médica Panamericana; 2005.

8. Utrera González A. "Hernia Umbilical". En: Cirugía de la pared abdominal. Guías clínicas de la Asociación Española de Cirujanos. $1^{\circ}$ Edición. Madrid: Arán; 2002.

9. Sociedad Argentina de Infectología. Guía para la Profilaxis Antibiótica Prequirúrgica. 2003 Agosto (fecha de acceso 9 de abril de 2005); 12. Disponible en www.sadi.org.ar

10. Muschaweek Ulrike. Umbilical and epigastric hernia repair. Surg Clin N Am 2003: 1207-1221.

11. Arroyo A, Pérez F, Serrano P. Is prosthetic umbilical hernia repair bound to replace primary herniorrhaphy in the adult patient? Hernia 2002; 6: 175-177.

12. Kurzer M, Belsham PA, Kark AE. Tension free mesh repair of umbilical hernia as a day case using local anaesthesia. Hernia 2004; 8: 104-107.

13. Menon VS, Brown TH. Umbilical hernia in adults: day case local anaesthetic repair. J Postgrad Med 2003; 49: 132-133.

14. Sanjay P, Reid TD, Davies EL. Retrospective comparison of mesh and sutured repair for adult umbilical hernias. Hernia 2005; 9: 248-251. 